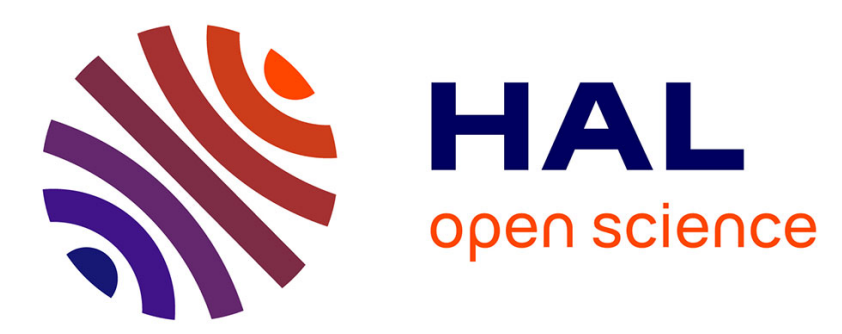

\title{
An Evidential Sensor Model for Velodyne Scan Grids
} Chunlei Yu, Véronique Cherfaoui, Philippe Bonnifait

\section{To cite this version:}

Chunlei Yu, Véronique Cherfaoui, Philippe Bonnifait. An Evidential Sensor Model for Velodyne Scan Grids. 13th International Conference on Control, Automation, Robotics and Vision (ICARCV 2014), Dec 2014, Singapore, Singapore. pp.583-588, 10.1109/ICARCV.2014.7064369 . hal-01098180

\section{HAL Id: hal-01098180 https://hal.science/hal-01098180}

Submitted on 23 Dec 2014

HAL is a multi-disciplinary open access archive for the deposit and dissemination of scientific research documents, whether they are published or not. The documents may come from teaching and research institutions in France or abroad, or from public or private research centers.
L'archive ouverte pluridisciplinaire HAL, est destinée au dépôt et à la diffusion de documents scientifiques de niveau recherche, publiés ou non, émanant des établissements d'enseignement et de recherche français ou étrangers, des laboratoires publics ou privés. 


\title{
An Evidential Sensor Model for Velodyne Scan Grids
}

\author{
Chunlei Yu, Véronique Cherfaoui and Philippe Bonnifait \\ Université de Technologie de Compiègne, \\ UMR CNRS 7253 Heudiasyc, \\ Compiègne, France \\ Email: \{chunlei.yu,veronique.cherfaoui, philippe.bonnifait\}@hds.utc.fr
}

\begin{abstract}
For the development of driving assistance systems and autonomous vehicles, a reference perception equipment including navigable space determination and obstacles detection is a key issue. The Velodyne sensor which provides high definition and omnidirectional information can be used for this purpose. Nevertheless, when scanning around the vehicle, uncertainty necessarily arises due to unperceived areas and noisy measurements. This paper proposes an inverse evidential model for the Velodyne in order to exploit its measurements in a 2D occupancy grid mapping framework. The evidential sensor model interprets the data acquired from the Velodyne and successively maps it to a Carthesian evidential grid using a fusion process based on the least commitment principle to guarantee information integrity. Experimental results prove that this approach can handle efficiently the uncertainties of the sensor and thus a highly reliable local reference map near the vehicle can be built for every timestamped perception system that needs evaluation or calibration.
\end{abstract}

\section{INTRODUCTION}

To cope with errors and uncertainties when building occupancy grid maps, Bayesian methods are the foundations of usual frameworks. Many extensions have been published in the literature, like the Bayesian Occupancy Filter (BOF) [1] which estimates both the occupancy and the speed of the cells. [2] proposed an extended occupancy grid approach which can be used to track non-rigid moving objects. [3] applied the bayesian occupancy grid map to detect road boundaries. [4] proposed novel forward model to interpret laser observations into occupancy grids. In this paper, we propose an evidential framework to build an occupancy grid map in the proximity of the host vehicle. The evidential theory [5] is used as the mathematical basis to build an inverse model. Indeed, by using this formalism, it is efficient to model state cell allocation and avoids introducing arbitrary a priori information in unoberved areas. The accumulation of scan echoes contributes to the detection of the ground and of the obstacles. Evidential grid mapping is not new. [6] built an evidential grid map using the data acquired from a sonar, [7] also adopted the evidential approach and built an evidential occupancy grid map in a Cartesian coordinates frame. Using the evidential framework to manage fusion is popular and [8] applied evidential fusion rules to manage sensor uncertainties.

The Velodyne lidar [9] provides rich and accurate information about the surrounding environment, an adapted sensor model to tackle its uncertainty and to fully profit its rich information is put forward. We propose a tailored sensor model which interprets the Velodyne data into local 2D occupancy grid maps. The least commitment principle is adopted here in order to avoid introducing any prior information. The model makes fully use of the evidential theory to handle the rich information provided by the Velodyne while keeping the processing load reasonable. A fusion process based on Dempster Shafer data fusion enhances the reference map by fusing data acquired at different locations.

The paper is organized as follows: section II presents the evidential framework, the theory basis will be illustrated. Section III details the main contribution of the work. An evidential sensor model is developed and utilized to merge high definition lidar measurements into scan grid maps. Section IV illustrates the fusion scheme based on the evidential framework. Section $\mathrm{V}$ shows the implementation details and experimental results.

\section{EVIDENTIAL FRAMEWORK}

In this framework, a frame of discernment $\Omega$ is defined to model the state of each cell. The frame of discernment is a finite discrete set, which contains all mutually exclusively propositions of interest. These basic propositions are called singleton propositions. In our case, the frame of discernment is defined as: $\Omega=\{O, F\}$, the two singletons are the proposition $O$ and the proposition $F$, indicating respectively that the specific cell is occupied and free. One has to increase this set, by considering the power set which is defined as $2^{\Omega}=\{\emptyset, F, O, \Omega\}$. With the frame of discernment, there are $2^{|\Omega|}$ possible subsets, where $|\Omega|$ is the cardinality of the set. Each subset is a possible proposition and in this manner it is possible to exhaustively propose other more general propositions based on interactions between the singleton propositions.

The meaning of each singleton proposition is detailed below:

- 1) $O$ indicates Occupied cell.

- 2) $F$ indicates Free cell.

- 3) $\Omega$ indicates ignorance about the state of the cell (Unknown cell).

- 4) $\emptyset$ indicates that no proposition fits the cell.

In evidential theory, different functions may be used to represent the information in the power set. One specific function which is mostly utilized in our approach is the basic probability assignment $(B P A)$, which is a direct support for a specific proposition. Let function $m$ denote a $B P A$ that maps each 
proposition in $2^{\Omega}$ to a numerical measure of direct support for that particular proposition. The function $m$ returns values in the range of $[0,1]$ and satisfies the conditions:

$$
\sum_{A \subseteq \Omega, A \neq \emptyset} m(A)=1, m(\emptyset)=0
$$

One powerful application of evidential theory is the fusion of different sources of information. Let $m_{1}$ and $m_{2}$ be two given mass functions describing the occupancy belief of the same cell. The result of the combination using Dempster's conjunctive rule is computed in the following way:

$$
\begin{gathered}
\left(m_{1} \oplus m_{2}\right)(A)=K \sum_{\forall B, C \in 2^{\Omega}, B \cap C=A, A \neq \emptyset} m_{1}(B) m_{2}(C) \\
K^{-1}=1-\sum_{\forall B, C \in 2^{\Omega}, B \cap C=\emptyset} m_{1}(B) m_{2}(C) \\
\left(m_{1} \oplus m_{2}\right)(\emptyset)=0
\end{gathered}
$$

In the mapping process, one inherent aspect is to merge sensor data from different locations at different time instants into the built map. This fusion process changes the space states from unknown (initial state) to another applicable state and enables to update and confirm the state of the cells. Typically, the Dempster's conjunctive rule is chosen as the fusion operator.

\section{From VElodyne Data to EVIDENTIAL SCAN GRID MAP}

In this section, an inverse sensor model for the Velodyne is developed. The sensor model has to be chosen with special attention since a well adapted model can greatly increase the perception performance. In our approach, we build a 2D evidential occupancy grid map with data from the Velodyne by making a projection on the ground plane. To develop the sensor model, we suppose that the ground is locally flat for every Velodyne scan.

\section{A. Polar sensor model basic concepts}

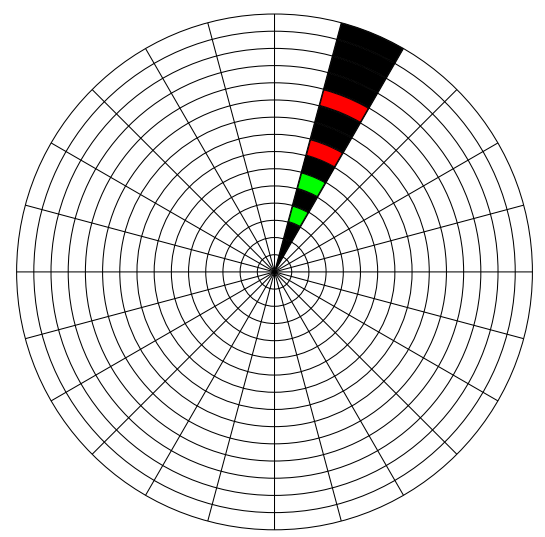

Figure 1: Space representation in Polar Coordinates, showing how measurements from Velodyne can be interpreted in the evidential framework. Green refers to free space, red refers to occupied space and dark refers to unknown space.
In order to be as close as possible to the sensor's rotating acquisition process, the scan grid map is created in a polar frame. As shown in Figure 1, the whole space around the car, which is placed at the origin, is divided into angular sectors, while each sector in the space is divided into different cells. For the BPA assignment process, we consider the sectors independent from each other. Indeed, if the sampling of the grid is high enough and since the laser beam width is very small, this assumption is well verified. In Figure 1, the colors represent different information. The dark cells mean that there is no information in the corresponding space due to an occlusion because of obstacles for instance. Green and red cells represent respectively Free and Occupied space.

This state assignment respects the least commitment principle. Velodyne points determine the state of the corresponding cells. Therefore, the space where there is no information is treated as Unknown which avoids adding wrong a priori information.

Although Velodyne is a high definition sensor, there might exist objects that are not detected. In Figure 2, six beams are drawn in the angular sector corresponding to angle $\lambda$, among which three hit the ground $(G 1, G 2, G 3)$, and the three other ones hit obstacles $(H 1, H 2, H 3)$. Due to the installation of the sensor on the roof, there is little information provided about the space between the host vehicle and the first impact on the ground, and between the consecutive impacts. As shown in the figure, there exists a non-null possibility that some nondetected objects exist in these areas, like $O 1, O 2, O 3$ and O4. Therefore, when projecting Velodyne data in $2 \mathrm{D}$, one needs to consider with attention what can happen between two consecutive beams. If the sensor is used for autonomous driving or driving assistance, this is crucial for safety.

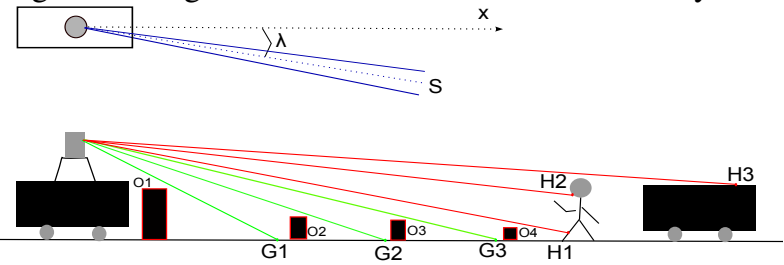

Figure 2: Some lidar beams in a particular angular sector $S$. Top, bird view of the host vehicle, $\mathrm{x}$ represents the motion direction. Bottom, lateral view of the scene.

\section{B. Thresholding the ground}

Let define an elevation threshold denoted $H$ which specifies the elevation of points considered as obstacles. The value of the threshold has to be chosen carefully in order to filter noise. When the elevation of an echo is above $H$, we consider the cell Occupied.

Figure 3 illustrates the state allocation process, and a specific sector is shown. To differentiate the ground information from the above-ground information, we set up a scene where a human and a car are near our host vehicle. Corresponding to the angular sector of angle $\lambda$, the lateral view is shown, where eight beams from Velodyne are drawn for illustrating purpose. The four lowest green beams hit the ground, where $G 1, G 2$, 


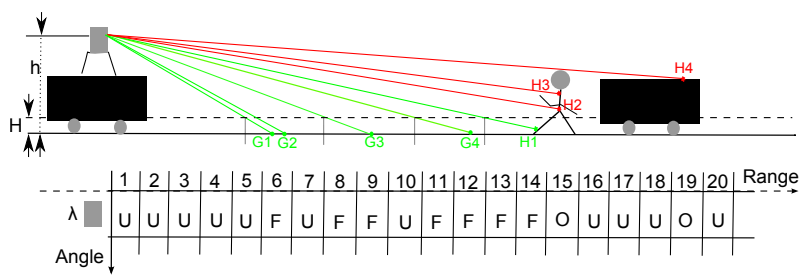

Figure 3: Sensor model for the Scan grid construction process. Top, lateral visualization of the threshold scene and of the backward free extrapolation (short vertical lines, explained in section III-C). Bottom, state assignment.

$G 3$ and $G 4$ are respectively the intersections of the signal and the ground. The four red beams reach the human and the car in the distance, and their intersections are $H 1, H 2, H 3$ and $H 4$. The grid in the bottom serves as an illustrating plot of the polar world model shown in Figure 3, in which the horizontal axis shows the range variation and the vertical axis represents the angle variation.

Based on the least commitment principle, the state allocation process obeys the following rules: As shown in Figure 3 , the cells which contain the $H 2, H 3$ and $H 4$ are marked $O$ (Occupied), as these points are above the threshold; the cells which contain respectively $G 1, G 2, G 3$ and $G 4$ are marked as $F$ (Free), as these points are detected on the ground; as depicted above, although $H 1$ is above the ground and hits on the person, the corresponding cell is allocated Free because it is under the threshold $H$. All the other cells are marked $U($ Unknown $)$.

\section{Backward free space propagation}

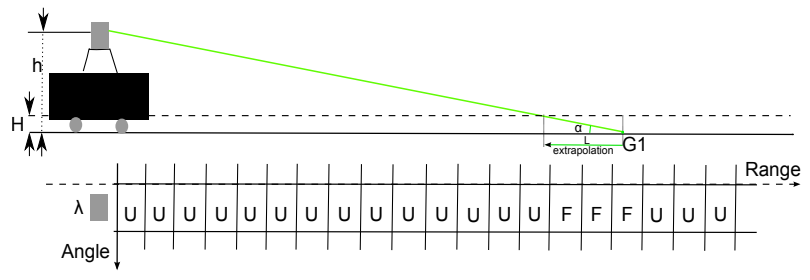

Figure 4: Backward extrapolation to the host vehicle: the two cells included in the interval $L$ are extended as Free space.

One benefit of defining the threshold $H$ is the extension of the Free region by making a backward extrapolation to the host vehicle. Figure 4 illustrates the principle: considering the beam which hits the ground, one can deduce that there is no obstacle in the interval $L$ which has an elevation superior to threshold $H$. In this case, the zone Free corresponding to $G 1$ is extended towards the host vehicle. Two more cells covered by the extension distance $L$ are set to Free. In Figure 3, the states of cell 8 cell 11 and cell 13 at angle $\lambda$ are also set to Free because we extrapolate at point $G 3, G 4$ and $H 1$.

\section{Eliminating conflicting impacts}

One conflicting situation might happen using the above sensor model, as shown in Figure 5, where the host vehicle detects one bus ahead, four beams are drawn for illustration. Based on the cell state allocation principle, the corresponding cells for $G 1$ and $G 2$ should be allocated Free, which conflicts with reality. Obviously, cell 10 should be allocated Occupied and cell 11 should be allocated Unknown based on the sensor readings. In our approach, we make an additional modeling: $a$ detected obstacle on the ground is modeled as a vertical surface that is linked to the ground. So in cells where both obstacle points and ground points are detected, ground points are ignored. This model brings the convenience of eliminating the conflicting cells, but at the expense of making impossible to differentiate hung obstacles from obstacles linked to the ground. The ground points detected behind the first obstacle surface are ignored and the corresponding cells rest Unknown until future exploration when the host vehicle moves.

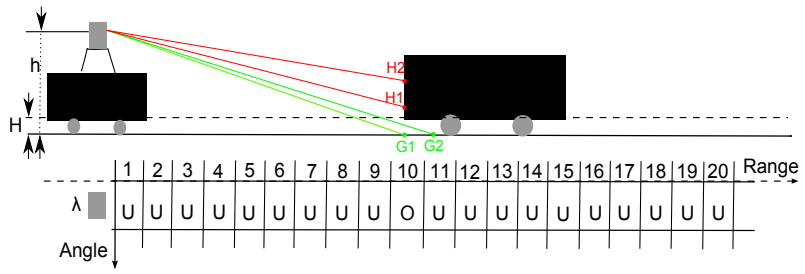

Figure 5: Conflicting information elimination

\section{E. Grid mass assignment}

We need now to assign a $B P A$ to the grid cells to quantify the belief. We propose a grid mass assignment model based on information accumulation. In Figure 3, cell 6, cell 9 and cell 12 are all set to Free. However, we should have unequal amount of beliefs about their Free state because in cell 6, there exist two points on the ground to support the state, whereas in cell 9 and cell 12, there exists only one. The same stands for the Occupied cells, cell 15 and cell 19 should have unequal amount of beliefs about the occupied state. More points supporting one state should contribute to more beliefs on the state. This accumulation concept reinforces the belief assigned to each proposition.

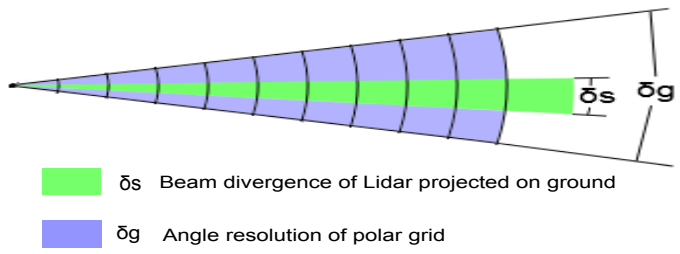

Figure 6: Missed-detection illustration

The $B P A$ values are based on sensor uncertainties. Let $\alpha_{F A}$ and $\alpha_{M D}$ correspond to the the probability of false alarm and missed-detection. A false alarm is when the sensor issues an impact whereas there is nothing. It depends essentially on the sensor noise and on multipath propagation. A missed detection is mainly related to the reflexivity of the target and to the ratio between the cell size and the beam width. Figure 6 shows how this ratio results in missed-detection. $\delta_{s}$ represents the divergence of Lidar (beam width), and $\delta_{g}$ represents the angular resolution of the polar grid. In this circumstance, one beam of Lidar can not cover the whole sector. This beam can 
miss potential obstacles within its blind regions of the cell. The missed-detection effect thus has to be considered.

The proposed model calculates the $B P A s$ with probabilistic approach. Based on the definition of false alarm, $\alpha_{F A}=$ $P\left(C=F \mid \xi_{1}\right)$, Where $\xi_{1}$ represents one obstacle impact in the cell, $C$ stands for the state of the cell. Supposing that errors are independent, the total false alarm probability in one cell given $n_{O}$ obstacle points are detected in this cell should be $P\left(C=F \mid \xi_{1}, \xi_{2}, \ldots, \xi_{N}\right)=\alpha_{F A}^{n_{O}}$. Thus the probability of Occupied can be represented as: $P\left(C=O \mid \xi_{1}, \xi_{2}, \ldots, \xi_{N}\right)=$ $1-\alpha_{F A}^{n_{O}}$. Based on the same methodology, for Free cells, the missed-detection probability $\alpha_{M D}=P(C=O \mid \Delta)$, where $\Delta$ represents no above ground impact is returned to the sensor. If we assume $n_{F}$ ground points are detected in this cell, the total missed-detection probability should be $\alpha_{M D}^{n_{F}}$. Thus the probability of Free should be represented as $1-\alpha_{M D}^{n_{F}}$.

Based on the principle, the $B P A$ assignment:

For a Free cell:

$m(O)=0, m(F)=1-\alpha_{M D}^{n_{F}}, m(\Omega)=1-m(F), m(\emptyset)=0$

For an Occupied cell:

$m(O)=1-\alpha_{F A}^{n_{O}}, m(F)=0, m(\Omega)=1-m(O), m(\emptyset)=0$

For an Unknown cell, the initial state is kept:

$$
m(O)=0, m(F)=0, m(\Omega)=1, m(\emptyset)=0
$$

To keep the processing load reasonable, we suggest to extrapolate the free level $m(F)$ uniformly with no decrease to the cells that have no echoes.

\section{F. From polar to cartesian}

The approach merges the Velodyne scan data into occupancy grid map. This map is built in polar coordinate system, but for the fusion purpose, we need to transform it into Cartesian coordinate system. All the information collected has to be transformed into Cartesian coordinates, with the least loss. Several methods exist to do the transform. In our approach, we have adopted the bilinear interpolation algorithm introduced by [10].

\section{Ego-MAP GRID Fusion SCHEME}

The scan grid map is not complete, because there exist uncertainties in the map due to unperceived space. The Unknown space between the host vehicle and the obstacles should be eliminated. With Dempster's conjunctive rule, the fusion of several successive scan grids allows to gradually eliminate the uncertainties in the map. In the proposed approach, a map is built by fusing the scan grids centered at different locations. To make this fusion, the ego-motion of the host vehicle has to be compensated and then every new scan grid of the Velodyne is merged into a grid denoted EgoMapGrid. Figure 7 illustrates the whole approach. The fusion process is sequential. At time $t$, the new scan grid ScanGrid $(t)$ updates EgoMapGrid $(t-1)$ to provide a new EgoMapGrid(t).

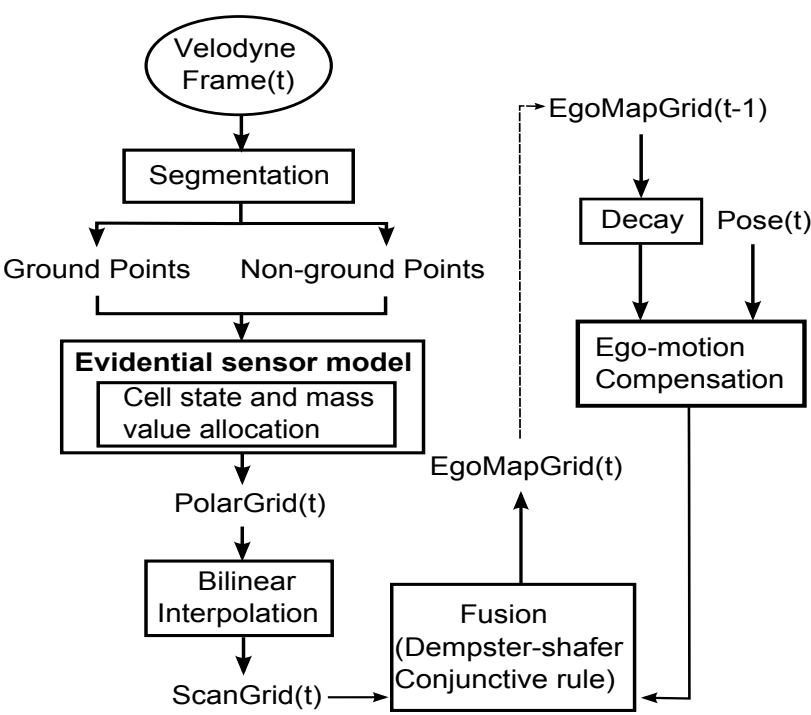

Figure 7: Workflow of the scan grid construction and fusion

To accommodate to the dynamic environment, we adopt the approach proposed by [11] and use a decay factor for EgoMapGrid. The information in EgoMapGrid can become aged and not consistent with reality. This effect can be especially important when moving objects are in the scene. Adopting decay factor means that all the information in the past will gradually become less evident. The equations below show how mass functions are discounted with a decay factor denoted $\beta$.

$$
\begin{gathered}
{ }^{\beta} m_{M}(A)=\beta * m_{M}(A), \quad A \subset \Omega \\
{ }^{\beta} m_{M}(\Omega)=1-\beta+\beta * m_{M}(\Omega)
\end{gathered}
$$

The fusion process adopts the Demster-Shafer conjunctive rule, as shown in Equation 1. For denotation purpose, let $m_{M, t}$ and $m_{S, t}$ represent respectively the mass functions of EgoMapGrid and ScanGrid at time $t$.

$$
m_{M, t}={ }^{\beta} m_{M, t-1} \oplus m_{S, t}
$$

\section{EXPERIMENTAL IMPLEMENTATION AND RESULTS}

\section{A. Experimental implementation}

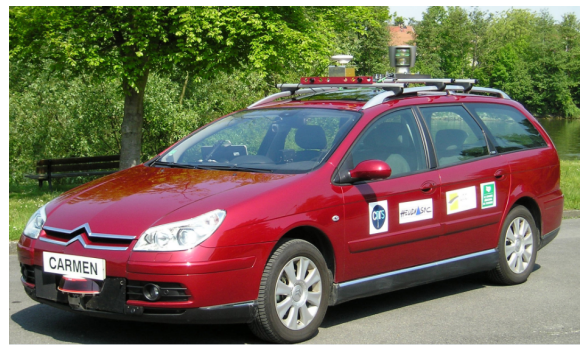

Figure 8: Experimental platform with the Velodyne sensor

The approach was tested with a vehicle of the experimental platform PACPUS shown in Figure 8. We have implemented the approach in $\mathrm{C}++$. In the approach, the Velodyne $H D L-$ $64 \mathrm{E}$ data was acquired at $10 \mathrm{~Hz}$ frequency. The segmentation process detects the ground plane and above ground points. We have adopted the ground labeling technique proposed by [12], which generally provides satisfactory results. The ego-motion between two scans is estimated using CAN data. 
For the purpose of demonstration, the scan grids of $(72 * 72)$ meters are built with uniform cells of $\operatorname{size}(0.1 * 0.1)$ meters. In the polar grid map, the angular resolution is 0.5 degrees and the radius resolution is 0.1 meters. For the tuning parameters, we have adopted $\alpha_{M D}=0.66, \alpha_{F A}=0.15 . \alpha_{M D}$ is based on the ratio of the beam divergence of Velodyne (estimated to 0.17 degrees by [13]) and the resolution of the grid (0.5 degrees). We have tuned empirically $\alpha_{F A}$ to 0.15 in order to consider the sensor noise and the multipath phenomenon.

\section{B. Results}

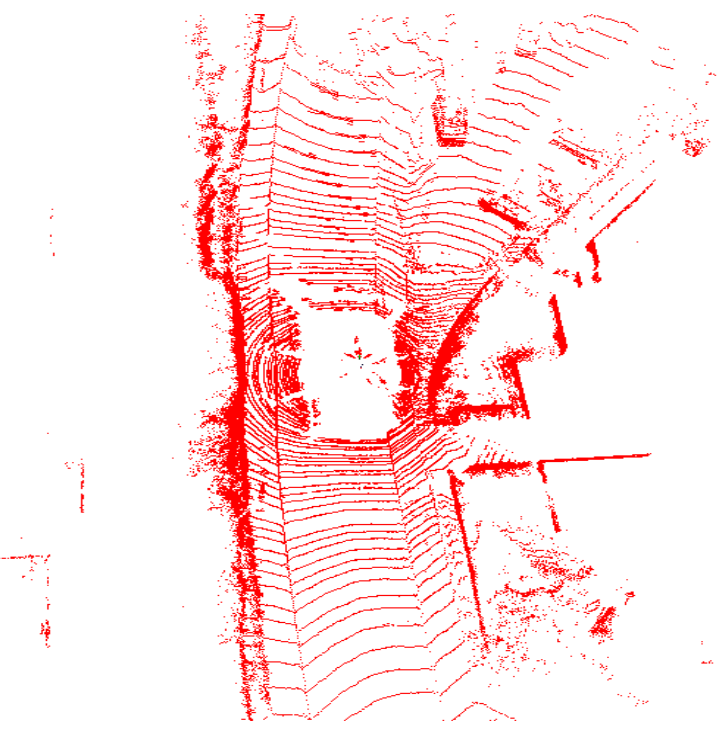

Figure 9: 3D display of the scene

For every instant, a Velodyne scan grid is built. For illustration of the system, we report in this section a typical scene where the host vehicle is in an urban road, as shown in Figure 9. The scene is depicted using Point Cloud Library $(P C L)$ [14]. Three occupancy maps are shown in Figure 10. Figure 10a shows result with no backward extrapolation while Figure $10 \mathrm{~b}$ and Figure 10c show scan grids with two different $H$ values for comparison, respectively $H=0.2$ and $H=0.4$. Conform to the sensor model, with a larger $H$ value, longer distance is extrapolated, so larger Free zones are observed in the grid map in which $H=0.4$. Augmentation of $H$ can reduce the uncertainties in the map, as less unperceived space is contained in the map thanks to backward extrapolation, but it also adds uncertainty to the system, because obstacles points with high elevation (but still beneath $H$ ) can be recognized as ground points. In reality, the vehicle can cross speed-bumpers or small slopes, with $H$ set appropriately, these situations pose no problem and the approach can be more robust.

The effect of information accumulation model is reflected by the green level, which corresponds to the Free mass degree. From the 3D display in Figure 9, the ground points received by Velodyne are typically denser in the space near the host system than farther away, which contributes to higher Free belief in the nearby space due to more ground points. Thus in the resultant grid map in 10c, the Free (green) space near the center is brighter than at the edge.

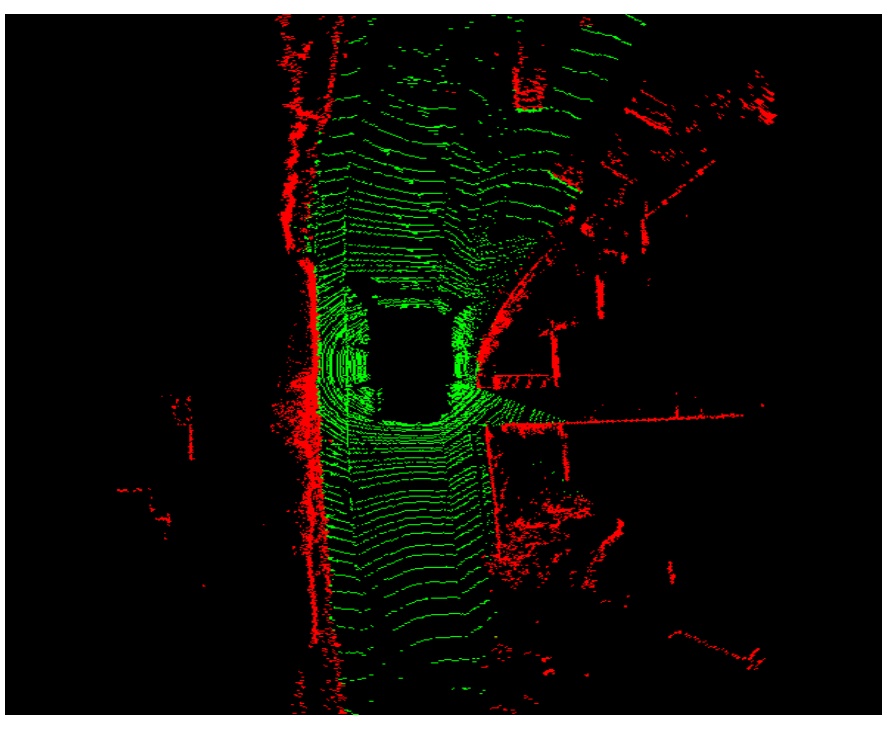

(a) Occupancy scan grid with no extrapolation

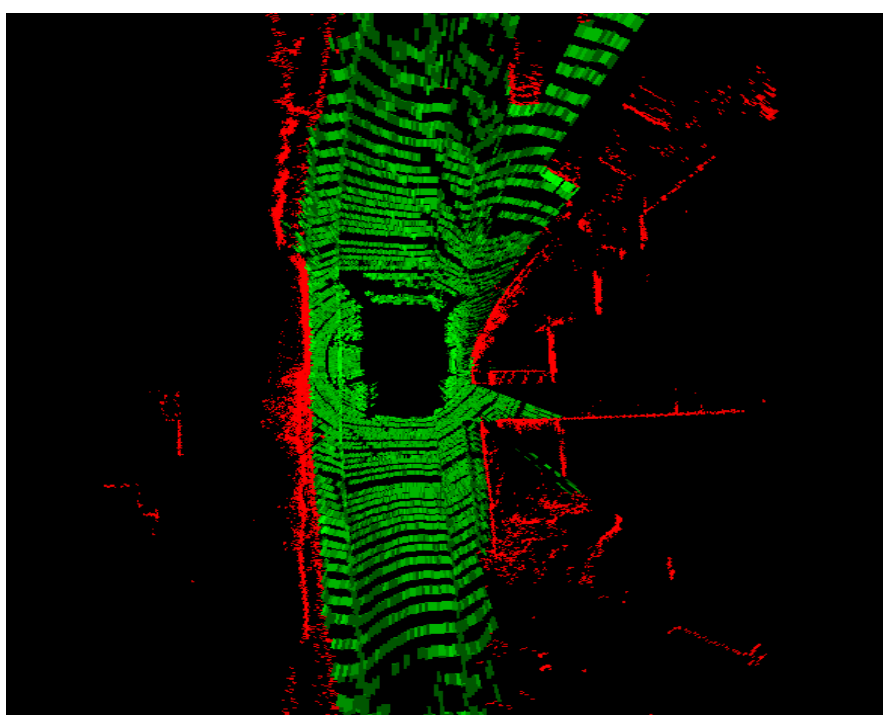

(b) Occupancy scan grid with backward extrapolation, $\mathrm{H}=0.2$

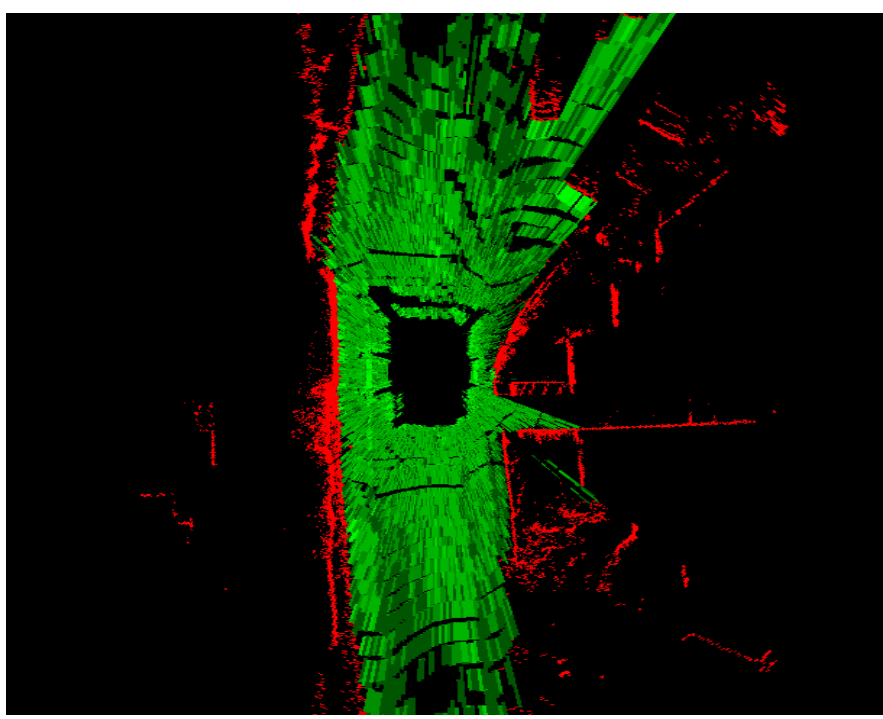

(c) Occupancy scan grid with backward extrapolation, $\mathrm{H}=0.4$

Figure 10: Scan Grids, Green represents Free space, Red represents Occupied space, Dark represents Unknown space. 
Figure 11 shows the fusion result. Corresponding to the scene depicted in Figure 9, $H$ is set to 0.2. One can remark that the green level in the central part of the fusion result map is lower compared to the other surrounding parts of the Free space. The reason is that the sensor receives no information from this space in the present scan. The resulting scan grid in Figure 10b shows that this space is Unknown, which makes no contribution to state confirmation in the EgoMapGrid, whereas the Free space surrounding this part accumulates in the Free mass from the ScanGrid, resulting to a higher Free mass degree in fusion.

The decay factor was set to 0.98 to slowly discount aged information. This effect is noticeable in the fusion result map shown in Figure 11a: the right bottom part of the map shows darker green which means less evidence to be Free. This can also be explained by the ScanGrid of Figure 10b: the state of this space is Unknown in the ScanGrid. With no evidence supporting the space state, the system tends to gradually forget its past state. In this case, $m(F)$ decreases until the system totally forgets the state, and it becomes Unknown again.

Figure 11b shows the result of the decision rule consisting in selecting the cell state which contains the maximum mass. This is the resultant reference map.

\section{CONCLUSION}

In this paper, we have proposed a new inverse sensor model to transform raw Velodyne data into local evidential grid maps. Based on the least commitment principle, the proposed model avoids introducing incorrect prior information in the grid state allocation of the space. The proposed way to fill the unobserved areas is based on the principle of information accumulation thanks to the fusion of successive scans taken at different points of view. This is performed by a spatiotemporal fusion process based on evidential theory. Based on the real experiments we have done to test the approach, we have observed that the approach provides good results in comparison to real scenes from a qualitative point of view.

\section{REFERENCES}

[1] C. Coue, C. Pradalier, C. Laugier, T. Fraichard, and P. Bessiere, "Bayesian occupancy filteing for multitarget tracking : an automotive application," International Journal of robotics research, vol. 25, no. 1, pp. 19-30, 2006

[2] B. Lefaudeux, G. Gate, and F. Nashashibi, "Extended occupation grids for non-rigid moving objects tracking," Intelligent Transportation Systems (ITSC), International IEEE Conference, p. 7, 2011.

[3] T. Weiss, B. Schiele, and K. Dietmayer, "Robust driving path detection in urban and highway scenarios using a laser scanner and online occupancy grids," Proceedings of IEEE Intelligent Vehicles Symposium, p. 6, 2007.

[4] K. Pathak, A. Birk, J. Poppinga, and S. Schwertfeger, "3d forward sensor modeling and application to occupancy grid based sensor fusion," IEEE/RSJ International Conference on Intelligent Robots and Systems, p. 6, 2007.

[5] G. Shafer, A Mathematical Theory of Evidence, P. U. Press, Ed. Princeton University Press, 1976.

[6] D. Pagac, E. M.Nebot, and H. Durrant-Whyte, "An evidential approach to map-building for autonomous vehicles," IEEE Transactions on Robotics, vol. 7, pp. 623-629, 1998.

[7] T. Yang and V. Aitken, "Evidential mapping for mobile robots with range sensors," IEEE Transactions on instrumentation and measurement, vol. 7, pp. 1422-1429, 2006.

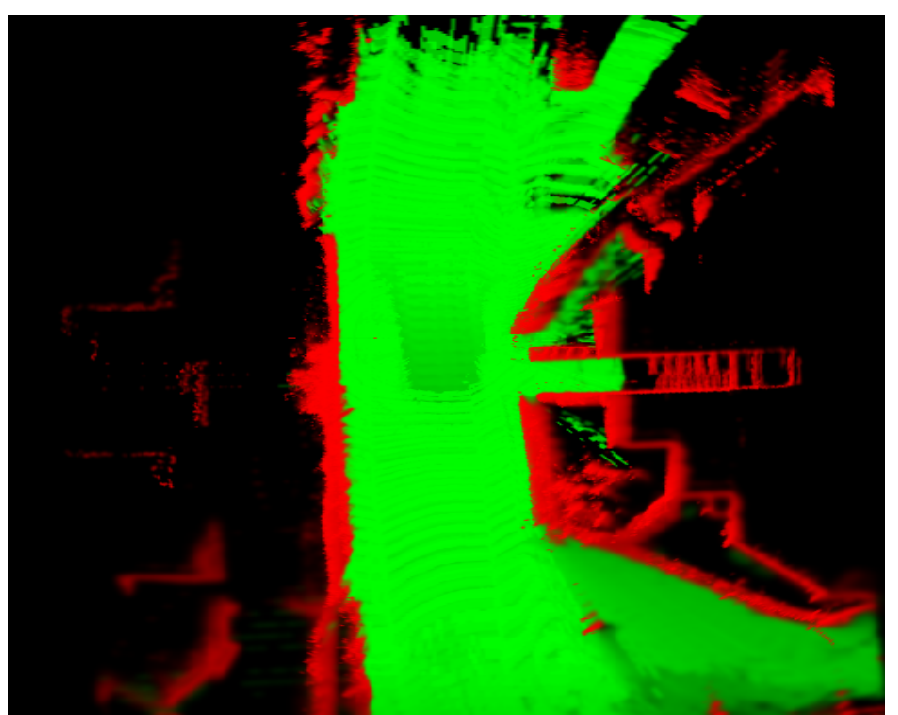

(a) Fusion result map of several scan grids.

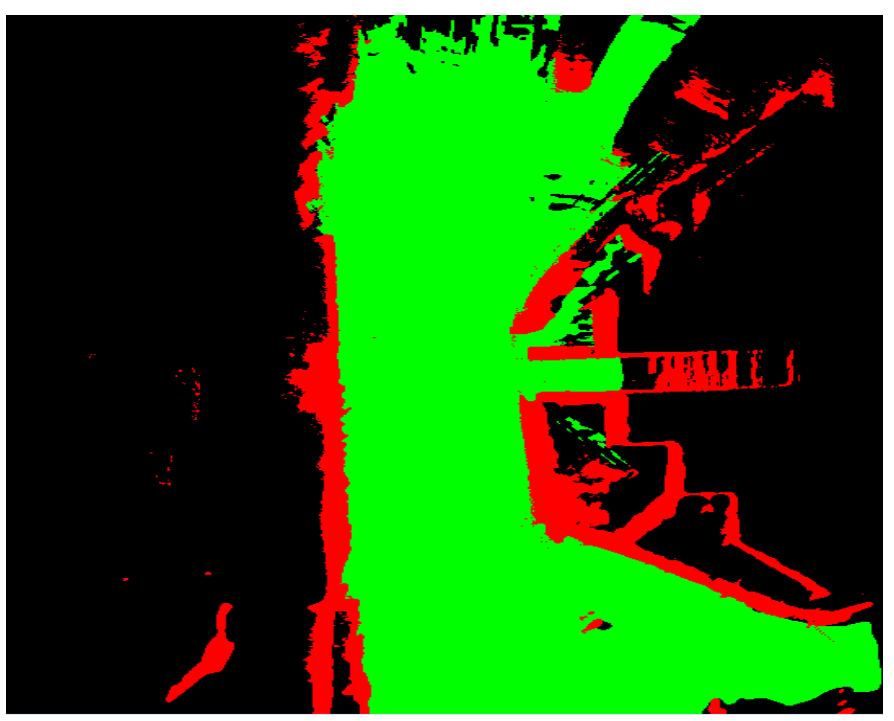

(b) Decision map deduced from fusion result map.

Figure 11: Fusion result

[8] F. Nashashibi, A. Khammari, and C. Laurgeau, "Vehicle recognition and tracking using a generic multi-sensor and multi-algorithm fusion approach," International Journal on Vehicle Autonomous Systems, 2006.

[9] V. L. Inc., HDL-64E S2 and S2.1 High Definition LiDAR Sensor User's Manual and Programming Guide. Velodyne LiDAR Inc., 2010.

[10] J. Moras, "Evidential perception grids for robotics navigation in urban environment," Ph.D. dissertation, Université de Technologie de Compiègne, 2013.

[11] J. Moras, V. Cherfaoui, and P. Bonnifait, "Moving objects detection by conflict analysis in evidential grids," IEEE Intelligent Vehicles Symposium (IV), vol. 6, 2011.

[12] A. Petrovskaya and S. Thrun, "Model based vehicle tracking for autonomous driving in urban environments," in IEEE International Conference Robotics and Automation (ICRA09). Workshop on Safe navigation in open and dynamic environments Application to autonomous vehicles. Kobe, Japan: IEEE, 2009.

[13] H. LONJARET and J. BENOIST, "Rapport d'essais de qualification de l'imageur laser," ANR, Tech. Rep., 2010.

[14] R. B. Rusu and S. Cousins, "3d is here: Point cloud library (pcl)," Robotics and Automation (ICRA), 2011 IEEE International Conference, vol. 4, 2011. 